https://doi.org/10.48009/2_iis_2006_246-251

\title{
THE DEVELOPMENT OF A RESEARCH AGENDA FOR RFID ADOPTION AND EFFECTIVENESS IN SUPPLY CHAINS
}

\author{
Vic Matta, Ohio University, matta@ohio.edu \\ Christopher Moberg, Ohio University, moberg@ohio.edu
}

\begin{abstract}
The use of newer information technologies, such as Radio Frequency Identification (RFID), can improve the speed, accuracy, and visibility of information exchange within supply chains, which can ultimately lead to reduced inventory costs and improved customer service [19]. In spite of all the attention being paid to RFID recently, the pace of actual or planned RFID adoption by firms in supply chains remains low to moderate [23]. In addition, given the positive hype of RFID often found in the trade press and at conferences, there is a surprisingly scarce number of conceptual and empirical research articles on RFID in the supply chain and technology literature streams. Therefore, the goal of this paper is to propose a research agenda on RFID adoption and effectiveness that is guided by previous adoption research of other technologies in business environments. This article proposes primary and secondary research designs to help managers by providing guidance on how to support RFID adoption. The primary design suggests confirming antecedents to adoption. The secondary design suggests checking for differences across different types of adopters.
\end{abstract}

Keywords: Supply Chain Technology, Logistics, RFID, Barcodes, Diffusion of Technology, Technology Adoption, Information Exchange

\section{INTRODUCTION}

Talluri defines the Supply Chain (SC) as a network of suppliers, manufacturers, distributors, and customers. Supply Chain Management (SCM) deals with the management and coordination of functions such as design, manufacture, distribute service, and recycle products or services [29].

In the past two decades many firms have been turning to SCM to lower costs, improve customer service, and to develop a sustainable competitive advantage [4]. For any firm interested in implementing SCM, one critical requirement is the exchange of operational and strategic information across functions within firms and between all firms that participate in the supply chain [19]. Further, in order for firms to fully take advantage of the benefits of information exchange, an investment in newer information technologies is required. Use of newer technologies to increase the speed, accuracy, and visibility of operational information exchange can lead to shorter cycle times, lower labor costs, and improved customer service [17]. These abilities are particularly important for the exchange of operational information among trading partners, such as point-ofpurchase sales and inventory levels [17].

Dröge and Germain define Electronic Data Interchange (EDI), as a business-to-business electronic ordering system that was the first IE enabling innovation in the supply chain [7]. From EDI and bar coding to the Internet and wireless communication equipment, many newer technologies have been introduced in the past thirty years that have supported information exchange. Radio Frequency Identification (RFID) is the latest technology to receive considerable attention in the trade press and among practitioners as a way to more accurately and efficiently manage inventory and exchange operational information. The ability of RFID to improve the efficiency of data collection and information exchange [16] makes RFID a significant technological innovation.

While the merits of RFID have been regularly discussed recently, surprisingly few firms in supply chains have adopted RFID technologies. For example, in a late 2004 warehousing study $15 \%$ of warehousing firms surveyed had adopted RFID, $41 \%$ firms were considering RFID adoption, and 44\% were not considering adoption at all [23]. The primary reason firms had adopted RFID was the recent mandate by Wal-Mart for its top suppliers to begin using RFID by January 2005 . Those firms not adopting RFID reported being unsure about the proposed benefits of RFID, particularly given the significant investment required [23].

Given the relatively low adoption of RFID to date and the corresponding scarcity of research in the literature to support those managers looking to adopt RFID, the goal of this paper is to provide a roadmap for future RFID research. After a brief review of the proposed benefits and challenges of RFID, the paper 
reviews barcodes and EDI adoptions. It points out that adoption may differ between coerced and freewill adopters. The paper will conclude with recommendations drawn from previous research streams on other technology adoptions such as EDI and barcodes. Two main research needs are identified-one that investigates and identifies antecedents of RFID adoption and the other that suggests the evaluation of these antecedents for differences between coerced and free-will adopters. This should provide managers with the additional leverage and guidance they need for successfully deploying RFID in their supply chains.

\section{On Barcodes and EDI}

It is important to note that RFID and barcodes have the same semantic functionality-that of machine readable identification. Both present a way of automatic identification of tagged items. The fundamental difference lies in the fact that barcodes require line of sight in order for them to be machine readable, whereas RFID tags do not. On the other hand, both innovations are largely integrated with EDI, though they can also be used independently. EDI typically uses internet protocols to communicate. The figure below shows that barcodes and EDI are typically integrated with EDI, and EDI typically uses the internet as a conduit for communications.

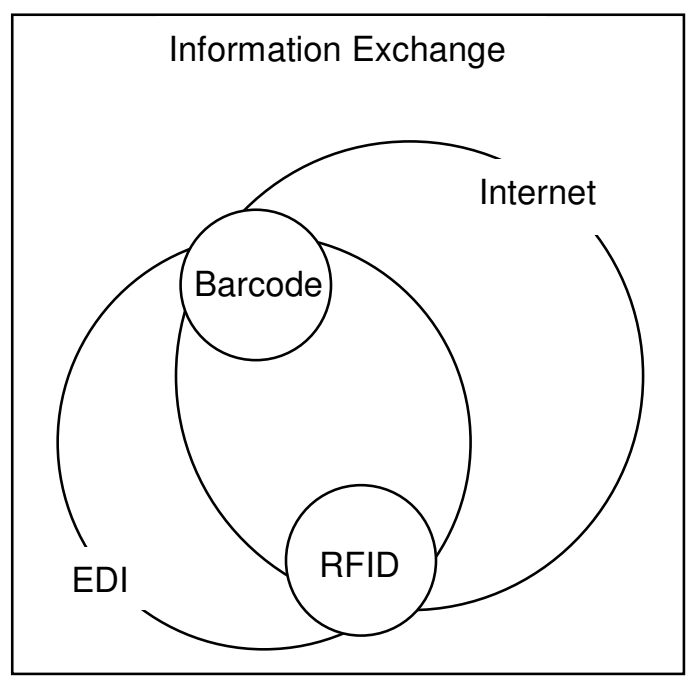

Figure 1. Relationships of IT Innovations in the Supply Chain

\section{Benefits and Challenges of RFID}

There are several proposed benefits of RFID, particularly as they relate to barcoding. First, the use of radio frequency technology allows readability from different angles, from a larger distance, and through materials without the need for line of sight as with barcodes. Furthermore, the tags contain a unique serial number and can withstand harsher conditions than barcodes. Such capabilities over barcodes extend operational efficiencies dramatically. The pervasive nature of RFID technology holds appeal for the manufacturing industry and supply chain sectors. Scanning RFID tags is easier because it does not require line of sight [23]. Ease of scanning facilitates inventory management and reduces the frequency of out-of-stock problems. Its ability to automate much of the record-keeping improves time management across the supply chain.

The Department of Defense (DoD) has been researching RFID for the purposes of tracking products as they traverse their supply chain, allowing high value goods to be tracked in transit. This has been called "In-Transit-Visibility" [13]. Another DoD initiative called "Total-Asset-Visibility" is described as the ability to see the condition, location, and inventory levels of products. These abilities of RFID to increase visibility of the tagged assets has been of interest to virtually all members of the supply chain.

Although there are compelling reasons to adopt RFID to replace barcodes, a few core issues exist: technology, standards, cost and privacy [14]. Technological implementations rarely go exactly as planned since new technology brings new challenges. Read ranges for the passive RFID tags are small (3 to 15 feet). Since RFID readers can read tags when pointed in the general direction of a tag, proximity reads can be an issue. Also, metals and liquids can distort/interfere with radio frequency signals, causing further loss in accuracy. In the past, development of open, internationally recognized standards have steered the barcode innovation to become mainstream. RFID systems are still developing common standards and protocols for use in the supply chain. While costs for readers and tags are becoming more affordable, the software development, integration and implementations, and supporting infrastructure costs are very high [14]. The risk of being outdated due to rapidly evolving standards makes the adoption less attractive.

From the social perspective, there is need for awareness and education. A common perception by consumers is that they could be tracked and monitored if they purchased something that contained an RFID tag [10]. Such a scenario is possible to create, but is far from practical and can only be done by authorized personnel. The RFID rollout needs to 
address this concern of perceived invasion of privacy [14].

\section{ADOPTION OF THE RFID INNOVATION IN THE SUPPLY CHAIN}

We discuss adoption of RFID from an innovation diffusion [25] perspective and juxtapose its adoption with that of its predecessors (EDI and barcodes) in the context of the supply chain.

At the most fundamental level, barcodes and RFID are automatic data collection tools for an information system. They enable and improve the efficiency of information exchange across the supply chain $[2 ; 21]$. In order to be effective, an information system needs to be standardized across organizations. One such inter-organizational information system that became a standard was EDI. EDI carried the data that was scanned from barcodes or (now) RFID tags [1; 32], as represented in Figure 1. The point to note here is that studies on barcodes invariably discuss EDI and do so in the context of the supply chain [1]. Therefore, adoption of barcode and RFID innovations are invariably discussed in the context of EDI and the supply chain.

\section{The Innovation of EDI and Barcodes}

Barcodes were the first form of automatic identification technology, providing advantages of reduced errors, increased speed, improved inventory management and enhanced communications $[1 ; 15$; 22; 27]. Seideman [26] traces the conception of barcodes innovation to a conversation that took place between Wallace Flint, president of a food chain and a dean at Drexel's Institute of Technology in 1948. Bernard Silver, then a graduate student at Drexel overheard Flint asserting the need for a system to automate the capture of product information at checkout. Silver collaborated with Woodland, another graduate student, beginning the first development of barcodes. In 1966, the National Association of Food Chains (NAFC) asked equipment manufacturers to speed up the process. RCA tested one of the first scanning systems at a Kroger store in Cincinnati in 1967. Then, in 1970 an industry consortium established an ad hoc committee to help develop and standardize barcodes. The first grocery application was demonstrated in 1971 and was standardized in 1972 by the Uniform Code Council (UCC) [11]. It was called the Universal Product Code (UPC).

There are two points of note here: first, the retail industry was the driving force for the adoption of barcodes; and secondly, adoption of this innovation was largely free willed and driven by the users [11].

\section{Coerced Adoption versus Free-Will Adoption}

Adoption of the barcode innovation may have largely been user driven and free willed. The users were the retail industry which had recognized a dire need for automatic data collection. In the retail industry, the first adopters of barcode technology were grocery stores, followed by home center retail (hardware, lumber and other small domestic construction, home repair and remodeling) companies. In a study on innovative automation technology in warehousing logistics spanning 1980 - 1988, Dadzie et al. [6] suggest that the warehouse industry wanted to use bar codes to improve productivity. These are signs of free will adoption.

A few signs of coerced adoption also have been found. While the first signs of UCC compliance mandates can be traced as far back as 1993 [5; 31], information about coerced adoption is scarce. One of the first coercive calls for compliance with standards comes from the automotive industry as recently as 2004 [9], in which Honda required their suppliers to comply with their EDI standards. More recently, U.S. and Canadian manufacturers were required to update their 12-digit UPC symbols with the new Sunrise 2005 standard of 13-digit UPC character system [33]. It appears that coerced adoption is a more recent phenomenon.

Walmart's mandate requiring their top hundred suppliers to use RFID tags at the palette level was followed by a similar mandate from Target and Tesco.[29]. This has created a large category of channel members who are coerced into adoption by power players in the supply chain. The point to note here is that free will adopters and coerced adopters will perceive different values for characteristics of the same innovation.

\section{Adoption of RFID}

Technology adoption has been researched along multiple dimensions: specific technologies, users perceptions, characteristics of adopters, etc. For example, some research has focused on adoption of spreadsheet software [3]. Innovation diffusion theory provides a useful perspective on how to improve technology assessment, adoption and implementation [8]. Within logistics, EDI technologies and their adoption have been examined [34]. Adoption of Logistics information systems were examined within channels. Reviews of other key studies help build a 
diffusion model applicable to the supply chain from the perspective of adoption of IT and more specifically, EDI. Williams and Rao [34] were able to explains adoption of automatic equipment identification (AEI) software among North American railroads using adoption theories (including diffusion). They examine users' perceptions of the innovation, the firm's culture, the types of communication channels used to diffuse knowledge of the innovation and various leadership factors. Moberg researches IE and posits that EDI and the Internet have drastically increased the speed and efficiency of IE. Since RFID is an IE enabler like the barcode, with only technological differences, his organizational postulates transfer to adoption of RFID as well. Moberg posits a positive relationship between top management commitment to newer technologies and both operational and strategic information exchange. He suggests that commitment to supply chain principles and the size (and therefore ability) of an organization to venture into the adoption of an innovation can also positively influence [16]. Russell and Hoag also use the diffusion of innovation theoretical framework to examine the social and organizational influences on adoption of IT [25]. Their model contributes factors that can be grouped under the perception of attributes of the innovation (such as relative or competitive advantage, compatibility of technology with existing systems, and complexity), and more organizational factors (such as centralization, level of connectivity, and openness of the system being proposed). Jeyaraj et al.'s survey article on adoption predictors [12] reviews 99 studies in IT adoption research and presents 135 independent variables. The full spectrum of diffusion is truly a composite of several of these characters. On superimposing the models discussed in our reviews from an organizational perspective, we found that different independent variables were classified along different dimensions. For example, Moberg classified his variables along environmental, organizational, and informational [16]. Russel and Hoag classified their independent variables along the constructs of perceived attributes, organizational factors, communication channels and leadership factors [25]. Jeyaraj's article lists a set of predictors that have been found most significant: perceived usefulness, management support and leadership, external pressure (peer and coercive), professionalism of the unit, and external information sources.

\section{CONCLUSIONS}

There is critical research needed to empirically link RFID adoption with the benefits proposed by academics and consultants. Too often, promising new theories or practices are not linked to tangible outcomes. For example, one common criticism of SCM theory development has been the lack of empirical research that connects SCM implementation with lower costs or improved customer service as suggested in the literature [18]. Recently, there have been several studies that examine the link between practice and outcomes that can be used to guide this second RFID research stream. For example, there has been research that examined the relationship of information exchange to supplier performance [28], information technology and warehousing performance [24], and the connection between SCM management components and logistics performance [20]. We need to find what kinds of adopters there are and what is important to them.

The next section addresses the agenda for research in confirming the short-listed variables and exploring differences between coerced and free-will adopters. Identifying what is important to different kinds of adopters will appropriately link benefits and impediments to coerced and free-will adopters.

\section{Proposed Research Agenda}

As is true with most technology adoptions, two opposing forces surround RFID adoption. One force is the tantalizing promise of potential benefits of RFID adoption. On the other hand, there is human resistance to technology adoption because it threatens many employees and because of the high costs of the new technology. Therefore, to overcome these, and other, barriers to adoption, research in two primary areas is needed. The first research need is to identify the antecedents of RFID adoption (see Table 1 for a summary of antecedents that were found significant) so that managers can develop strategies to increase the speed of adoption within supply chains.

It is important to note that adoption of technological innovations has been studied previously on other technologies such as computers, software, and EDI [35]. Using the studies listed in the previous section, we have classified variables that are significant and relevant to adoption of the RFID innovation. We propose a research agenda to first confirm the influence of the adoption variables listed in Table 1. Once those have been identified, managers can make the appropriate strategy decisions that support RFID adoption by individuals within organizations.

Once the factors that significantly influence the adoption of RFID have been validated, as a second 
phase it would be helpful to explore how these values differ across the supply chain between free-will adopters, coerced adopters, and non adopters.

Table 1. Categories of Technology Adoption Variables

\begin{tabular}{|c|c|c|}
\hline No & & aracteristic \\
\hline \multirow{5}{*}{1} & \multicolumn{2}{|c|}{ External influences $[12 ; 24]$} \\
\hline & & Coercive pressure \\
\hline & B & Peer pressure \\
\hline & $\mathrm{C}$ & External information sources \\
\hline & $\mathrm{D}$ & Competitive and relative advantage \\
\hline \multirow[t]{5}{*}{2} & \multicolumn{2}{|c|}{$\begin{array}{l}\text { Perceived professional benefits } \\
{[12 ; 25 ; 26 ; 35]}\end{array}$} \\
\hline & \multicolumn{2}{|r|}{$\begin{array}{l}\text { Simplicity and the integratability with } \\
\text { A existing systems }\end{array}$} \\
\hline & \multicolumn{2}{|r|}{$\begin{array}{l}\text { Quality of service and product provided } \\
\text { B by the RFID system }\end{array}$} \\
\hline & \multicolumn{2}{|c|}{ C Return on investment and initial cost } \\
\hline & \multicolumn{2}{|c|}{$\begin{array}{ll} & \text { Observability, } \\
\text { D } & \text { RFID system }\end{array}$} \\
\hline \multirow[t]{6}{*}{3} & \multicolumn{2}{|c|}{$\begin{array}{l}\text { Organizational Characteristics } \\
{[12 ; 17 ; 26]}\end{array}$} \\
\hline & A & IT commitment \\
\hline & B & Organizational size \\
\hline & $\mathrm{C}$ & $\begin{array}{l}\text { User involvement, participation, } \\
\text { training and support }\end{array}$ \\
\hline & $\mathrm{D}$ & Top management support \\
\hline & E & Leadership and championship in-house \\
\hline
\end{tabular}

Such an understanding of the system of values held by adopter groups would better prepare decision makers with the comprehension of issues at stake. It would allow RFID implementations to be more practical, better planned, and better matched to the needs of the supply chain entities.

Research that examines the adoption of RFID technologies and the differential values across the adopter groups-and whether it is significantly and positively related to improved accuracy, lower labor, stock-out and inventory costs, and increased visibility of in-transit orders and inventory-is critical. If it can be empirically demonstrated to the coerced adopters that the performance of those firms that have adopted RFID has improved, it will increase the likelihood that managers can overcome the resistance to the cost and risk of RFID adoption that is so common in today's supply chain environment.

\section{REFERENCES}

1. Bartko, P. W. (1996). EDI and barcoding converging. EDI World, 6, 6.

2. Beamon, B. M. (1998). Supply chain design and analysis: Models and methods. International Journal of Production Economics, 55(3), 281294.

3. Brancheau, J. C., \& Wetherbe, J. C. (1990). The adoption of spreadsheet software: Testing innovation diffusion theory in the context of enduser computing. Information Systems Research, 1(2), 115-143.

4. Brewer, P. C., \& Speh, T. W. (2000). Using the balanced scorecard to measure supply chain performance. Journal of Business Logistics, 2l(1), 75-94.

5. Cooke, J. A. (1993). Bar code or perish. Traffic Management, 32, 32-35.

6. Dadzie, K. Q., \& Johnston, W. J. (1991). Innovative automation technology in corporate warehousing logistics. Journal of Business Logistics, 12(1), 63-82.

7. Dröge, C., \& Germain, R. (2001). The relationship of electronic data interchange with inventory and financial performance. Journal of Business Logistics, 21(2), 209-230.

8. Fichman, R. G. (1992). Information technology diffusion: A review of empirical research. Paper presented at the International Conference on Information Systems.

9. Fulcher, J. (2005). Honda sets a higher standard for EDI compliance. Manufacturing Business Technology, 23, 1.

10. Gilbert, A. (2003). Major retailers to test smart shelves. News.Com, 2005, from http://news.com.com/Major+retailers+to+test+s mart+shelves/2100-1017_3-979710.html?tag=nl

11. Haberman, A. L. (2001). Twenty-five years behind bars: The proceedings of the twenty-fifth anniversary of the U.P.C. At the Smithsonian institution, September 30, 1999 / Alan l. Haberman, general editor (Vol. Preface). Cambridge, Mass.: Harvard University Wertheim Publications Committee: distributed by Harvard University Press.

12. Jeyaraj, A., Rottman, J. W., \& Lacity, M. C. (2006). A review of the predictors, linkages, and biases in it innovation adoption research. Journal of Information Technology, 21, 1-23.

13. Kolleda, D. (2005). Achieving in-transit visibility (ITV): A study of technology on ITV in the department of defense: U.S. Army War College,Carlisle Barracks, Carlisle, PA,170135050 . 
14. Lundstrom, S. (2004). RFID: Just the facts: AMR Research. Retrieved 2006 from http://www.idii.com/wp/rpRFIDJustTheFacts.pdf

15. Mack, S. L. (1998, Feb 98). Making a read on bar codes. Managing Office Technology Epnet Retrieved 2006 from http://search.epnet.com/login.aspx?direct=true \& $\mathrm{db}=$ buh\&an $=258762$

16. Moberg, C. R., Cutler, B., Gross, A., \& Speh, T. (2002). Identifying antecedents of information exchange within supply chains. International Journal of Physical Distribution and Logistics Management, 32(9), 755-770.

17. Moberg, C. R., \& Speh, T. (2003). Evaluating the relationship between questionable business practices and the strength of supply chain relationships. Journal of Business Logistics, 24(2), 1-19.

18. Moberg, C. R., Speh, T., \& Freese, T. (2003). Supply chain management: Making the vision a reality. Supply Chain Management Review, 7(5), 34-39.

19. Moberg, C. R., Whipple, T., Cutler, B., \& Speh, T. (2004). Do the management components of supply chain management affect logistics performance? International Journal of Logistics Management, 15(2), 15-31.

20. Niven, P. (2006). Glossary of key performance management. Paul Niven's Balanced Scorecard Academy QPR Software, PLC Retrieved 2006, 2006, from http://www.balancedscorecard.biz/Glossary.pdf

21. Nixon, K. (1996). Bar code basics. EDI World, 6, 32-34.

22. Osyk, B., \& Vijayaraman, B. S. (2005). RFID in the warehousing industry: Where do WERC members stand? Chicago, Illinois: Warehousing Education Research Council.

23. Rogers, D. S., Daugherty, P. J., \& Ellinger, A. E. (1996). The relationship between information technology and warehousing performance. The Logistics and Transportation Review, 32(4), 409-421.
24. Rogers, E. (2003). Diffusion of innovations: Free Press.

25. Russell, D. M., \& Hoag, A. M. (2004). People and information technology in the supply chain. International Journal of Physical Distribution \& Logistics Management, 34(2), 102-122.

26. Seideman, T. (1993). Bar codes sweep the world. Invention and Technology Magazine Retrieved June, 2006, 2006, from

http://www.americanheritage.com/articles/magaz ine/it/1993/4/1993_4_56.shtml

27. Stank, T. P., Daugherty, P. J., \& Ellinger, A. E. (1996). Information exchange, responsiveness, and logistics provider performance. The International Journal of Logistics Management, 7(2), 43-57.

28. Sullivan, L. (2004, 9/27/2004). Walmart's way. InformationWeek, 36-50.

29. Talluri, S. (2000). An it/is acquisition and justification model for supply-chain management. International Journal of Physical Distribution \& Logistics Management, 30(3/4), 221-237.

30. Unknown. (1994). Handbook available on barcode compliance. Traffic Management, 33, 1.

31. Unknown. (1998, Jan1998). EDI integrated with bar code system. Automotive Manufacturing \& Production, 110.

32. Unknown. (2004). 2005 Sunrise. Uniform Code Council, 2006, from http://www.uccouncil.org/ean_ucc_system/stnds_and_tech $/ 200$ 5\%20Sunrise\%20Flyer\%20008698.pdf

33. Walton, L. W. (1994). Electronic data interchange (EDI): A study of its usage and adoption within marketing and logistics channels. Transportation Journal, 34(1), 37-46.

34. Williams, L. R., \& Rao, K. (1998). Information technology adoption using classical adoption models to predict AEI software implementation. Journal of Business Logistics, 19(1), 5-16. 